\title{
Comparison between 2, 4 and 6 Minute Sitting Positions versus Immediately Lying Down on Hemodynamic Variables, Fluid and Ephedrine Requirement after Spinal Anesthesia in Elective Cesarean Section
}

Gamal Farook Mohamed Amer, El-Desoky Mohamed Ibraheem Nouh, Ali Abdel El moteleb Ali Ali Department of Anesthesiology and Intensive Care, Faculty of Medicine, Al-Azhar University

Corresponding author: Ali Abdel El moteleb Ali Ali, Mobile: 01148518954

\begin{abstract}
Background: hypotension is one of the most common complications of spinal anesthesia in patients undergoing cesarean section. In this regard, the patient's position may affect the incidence of hypotension.

Objective: to compare the incidence of hypotension and ephedrine requirement after spinal anesthesia with variable time in sitting position before lying down.

Patients and Methods: a total of 100 patients, scheduled for cesarean section under spinal anesthesia, with $10 \mathrm{mg}$ of hyperbaric bupivacaine $0.5 \%(2 \mathrm{ml})$ mixed with $25 \mu \mathrm{g}(0.5 \mathrm{ml})$ fentanyl, were randomly divided into 4 groups $(25$ patients per group). Groups M2, M4 and M6 remained in a sitting position for 2, 4 and 6 minutes after the induction of spinal anesthesia, respectively, while group M0 was immediately placed in a lying position.

Results: there were highly significant differences between the four groups as regard the incidence of hypotension based on Chi-square test; **p-value <0.001 HS. The frequency of hypotension was higher in group M0 in comparison with other groups. There was high consumption of ephedrine and fluid in group M0 than other groups as the total dose used in group M0 was bigger than other groups while M6 had the smaller dose.

Conclusion: the present study revealed that the patient's position is an important factor, which affects the frequency of hypotension, ephedrine and fluid requirements, and the onset of sensory block during the administration of spinal anesthesia for cesarean section.
\end{abstract}

Keywords: Systolic blood pressure, Spinal anesthesia, Ephedrine, Sitting position.

\section{INTRODUCTION}

Spinal anesthesia has been regarded as a reasonable anesthetic option for cesarean delivery since 1977. The advantages of this technique include avoidance of airway complications and depressant agents, as well as the mother's ability to remain awake and enjoy the birthing experience ${ }^{(1)}$.

Spinal anesthesia is frequently accompanied by hypotension, which may be defined in absolute terms as a systolic blood pressure (SBP) $\leq 90$ or in relative terms as a percentage (20\% fall from baseline). The incidence of hypotension can be as high as $70-80 \%$ when pharmacological prophylaxis is not used, the severity depends on the height of the block, the position of the parturient and whether it is elective or emergency cesarean section ${ }^{(2)}$.

Several strategies, including uterine displacement, lower legs compression and the administration of fluids and vasopressors, have been shown to decrease the occurrence of this hypotension however, none has shown to eliminate the need to treat it. In contrast, patient position has had variable effects on the incidence of hypotension after spinal anesthesia ${ }^{(3)}$.

Prevention of aortocaval compression is universally recommended to prevent hypotension and avoid the risk of an abrupt fall in venous return with subsequent collapse of cardiac output (CO) and blood pressure (BP). It can be achieved by using a wedge under the right hip or lumbar region, by tilting the table or by manually displacing the uterus to the left side ${ }^{(4)}$.

Maternal position may affect the speed of onset of sensory block and thus the hemodynamic effects ${ }^{(5)}$.
Therefore, allowing the patient to remain in the sitting position, instead of immediately lying down, could delay the spread of anesthesia and reduce the incidence of hypotension.

\section{AIM OF THE WORK}

This randomized prospective control study aim to compare the incidence of hypotension and ephedrine requirement after spinal anesthesia with 10 $\mathrm{mg}$ of hyperbaric bupivacaine $0.5 \%(2 \mathrm{ml})$ mixed with $25 \mu \mathrm{g}(0.5 \mathrm{ml})$ fentanyl in 4 groups. Groups M2, M4 and M6 remained in a sitting position for 2, 4 and 6 minutes after the induction of spinal anesthesia, respectively, while group M0 (control group) was immediately placed in a lying position.

\section{PATIENTS AND METHODS}

Setting: The study carried out in Al-Azhar University Hospitals.

Ethical Considerations: After approval of The Institutional Ethics Committee of The faculty of Medicine, Al-Azhar University; 100 patients scheduled for elective cesarean section under spinal anesthesia were enrolled in this prospective randomized study. All patients were counseled for the study protocol and a written informed consent was obtained from study participants.

Inclusion criteria: Age 18-45 years old. ASA I and II with gestational age of $>37$ weeks. Elective cesarean section. Height from $155 \mathrm{~cm}$ to $175 \mathrm{~cm}$ and Weight from $60 \mathrm{~kg}$ to $110 \mathrm{~kg}$. 
Exclusion criteria: Patient refused spinal anesthesia. Patient who had allergy to drugs used in the study. Preeclampsia patient. Chronic hypertension or diabetes. Contraindication to neuro axial anesthesia. Blood pressure below $90 \mathrm{mmHg}$ and heart rate (HR) below 50/min. Addiction or drug abuse. Contraindication to general anesthesia.

Methods of randomization: Randomization of patients was done using a computerized program. Packing, sealing and numbering of envelops was performed by a medical personnel (Under the supervision of doctors from the Department of Anesthesiology). The number of cases included in this study was randomly allocated into four groups. The sample size was calculated based on the study about effects of sitting up for five minutes versus immediately lying down after spinal anesthesia for cesarean delivery on fluid and ephedrine requirement (6). Finally, a total of 25 women were sufficient and included in each group.

Materials: Hyperbaric bupivacaine $0.5 \%$ and fentanyl. Monitor, pulse oximetry, NIBP, ECG. Ringer`s fluid infusion. Emergency drugs. Resuscitation equipments and drugs. Ranitidine 50 $\mathrm{mg}$ and ondansetron $4 \mathrm{mg}$. Atropine and Ephedrine.

Study groups: 4 Groups, 25 patients each. They all received $10 \mathrm{mg}$ of hyperbaric bupivacaine $0.5 \%$ (2 $\mathrm{mL})$ mixed with $25 \mu \mathrm{g}(0.5 \mathrm{ml})$ of fentanyl in induction of spinal anesthesia at the L3-L4 interspace, while the subject in a sitting position.

M0 (Control) patients were immediately lying down. Group M2 (25) patients sat for 2 minutes before lying down.

Group M4 (25) patients sat for 4 minutes before lying down.

Group M6 (25) patients sat for 6 minutes before lying down.

Anesthetic techniques: All patients were medically checked in the preoperative assessment clinic \{history, physical examination, investigations (e.g. complete blood picture, coagulation profile, liver and kidney functions and FBG).

Patients monitoring: Pulse oximetry. ECG. Noninvasive blood pressure.

Premedication: Patients fasted 8 hours according to fasting guidelines and received $4 \mathrm{~m} \mathrm{~g}$ of I.V ondansetron 1 hour before arrival in the operating room in addition to antacid prophylaxis in the form of ranitidine $50 \mathrm{mg}$ I.V.

Induction: After patient arrival to operating room, patient placed in $15^{\circ}$ left tilted supine position and I.V catheter $20 \mathrm{G}$ was inserted and $500 \mathrm{~mL}$ of Ringer's solution was infused over 15 minutes, and base line parameters were recorded.
Spinal anesthesia was induced by a 25 -gauge spinal needle at the L3-L4 interspace, while the subject in a sitting position. Spinal anesthesia consisted of $10 \mathrm{mg}$ of hyperbaric bupivacaine $0.5 \%$ $(2 \mathrm{~mL})$ mixed with $25 \mu \mathrm{g}(0.5 \mathrm{ml})$ fentanyl was administered over 12 seconds after sterilization of the back of the patient with povidone-iodine $10 \%$ (betadine).

All participants received supplemental oxygen $(2-3 \mathrm{~L} / \mathrm{min})$ via face masks. Systolic blood pressure (SBP), HR and mean arterial blood pressure (MAP) were recorded 1 minute before (baseline) and 1,3 , and 5 minutes after the induction of spinal anesthesia. Thereafter, measurements were performed in 5-minute intervals till the end of the surgery. Adverse effect (i.e., nausea, vomiting, pruritus, or bradycardia) along with the subject's need for ephedrine, atropine, or a rescue analgesic, were recorded.

Sensory block level was assessed (based on loss of sensation to hot and cold test) immediately after the subjects reclined and every 1 minute thereafter until she reached constant sensory level. The measured time was recorded as the onset of sensory block up to T6, and at this time, surgery was allowed.

A modified Bromage scale was recorded 1 and 3 minutes after induction of the spinal anesthesia (3, no movement; 2 , only able to flex the ankle and foot; 1 , able to bend the knee; and 0 , no paralysis and able to raise the extended leg). Hypotension (SBP $\leq$ $90 \mathrm{mmHg}$ or $>20 \%$ decline from the base line) was treated with $5 \mathrm{mg}$ of IV ephedrine bolus; this dose was repeated as necessary to achieve an SBP of $\geq 90$ mmHg.

Additionally, if the mother's HR $<50$ beat $/ \mathrm{min}, \quad 0.5 \mathrm{mg}$ of IV atropine bolus was administered. Then, $20 \mathrm{IU}$ of oxytocin was infused in $500 \mathrm{~mL}$ of Ringer's solution after delivery. If the parturient complained of pain at any time during surgery, $50 \mu \mathrm{g}$ of IV fentanyl was administered. If the analgesia was inadequate, the mentioned dose was repeated and if failed conversion to general anesthesia was proceed. Upon the occurrence of nausea or vomiting, SBP was checked. In case it is $\leq$ $90 \mathrm{mmHg}$, treatment with $5 \mathrm{mg}$ of IV ephedrine was given otherwise, treatment with $4 \mathrm{mg}$ of ondansetron IV.

\section{The following variables were recorded:}

HR (beats/min), SBP, DBP, and MBP ( $\mathrm{mmHg}$ ) were recorded 1 minute before (baseline) and 1,3 , and 5 minutes after the induction of spinal anesthesia. Thereafter, measurement was performed in 5-minutes intervals till the end of the surgery. Onset and offset of sensory and motor block. Level of sensory block up to T6. Fluid, ephedrine and atropine requirements. 
Apgar score at 1 and 5 minute after delivery. Estimation of blood loss and urine output.

\section{Statistical analysis}

Recorded data were analyzed using the statistical package for social sciences, version 20.0 (SPSS Inc., Chicago, Illinois, USA). Quantitative data were expressed as mean \pm standard deviation (SD). Qualitative data were expressed as frequency and percentage.

The following tests were done: A one-way analysis of variance (ANOVA) when comparing between more than two means. Post Hoc test: Least Significant Difference (LSD) was used for multiple comparisons between different variables. Chi-square $\left(\mathrm{x}^{2}\right)$ test of significance was used in order to compare proportions between qualitative parameters. The confidence interval was set to $95 \%$ and the margin of error accepted was set to $5 \%$. So, the p-value was considered significant as the following: Probability (P-value): P-value $<0.05$ was considered significant. $\mathrm{P}$-value $<0.001$ was considered as highly significant. $\mathrm{P}$-value $>0.05$ was considered insignificant.

\section{RESULTS}

There were no significant differences among the four groups with respect to demographic data (age, weight, height, gestational age, and ASA), and the duration of surgery as in table (1)

Table (1): Comparison between groups according to demographic data.

\begin{tabular}{|c|c|c|c|c|c|}
\hline Demographic Data & M0 (n=25) & M2 (n=25) & M4 (n=25) & M6 (n=25) & $\begin{array}{r}\text { P- } \\
\text { valu }\end{array}$ \\
\hline Age (years) & $18-45$ & $18-45$ & $18-45$ & $18-45$ & \\
Range & $32.13 \pm 7.07$ & $33.74 \pm 7.42$ & $31.81 \pm 6.93$ & $33.40 \pm 7.27$ & $>0.05$ \\
Mean \pm SD & $78.12 \pm 5.47$ & $82.03 \pm 5.74$ & $77.34 \pm 5.36$ & $81.21 \pm 5.63$ & $>0.05$ \\
\hline Weight (kg) & $162.74 \pm 9.39$ & $160.88 \pm 11.96$ & $161.11 \pm 12.16$ & $160.17 \pm 10.72$ & $>0.05$ \\
\hline Height (cm) & $38.12 \pm 0.76$ & $38.50 \pm 0.80$ & $37.74 \pm 0.75$ & $38.12 \pm 0.78$ & $>0.05$ \\
\hline Gestational age (wks) & & & & & \\
\hline Operating time (min) & $45-60$ & $45-60$ & $45-60$ & $45-60$ & $>0.05$ \\
Range & $53.03 \pm 6.36$ & $55.68 \pm 6.68$ & $52.49 \pm 6.24$ & $56.17 \pm 6.55$ & \\
Mean \pm SD & $18(72 \%)$ & $17(68 \%)$ & $16(64 \%)$ & $18(72 \%)$ & $>0.05$ \\
\hline ASA & $7(28 \%)$ & $8(32 \%)$ & $9(36 \%)$ & $7(28 \%)$ & \\
I & & &
\end{tabular}

There were statistically significant difference between group M0 and other groups (M2, M4, and M6) as regard systolic blood pressure, diastolic blood pressure, and mean arterial pressure at 1,3, 5, 10, and 15 minutes after induction of spinal anesthesia as in tables (2,3 and 4).

Table (2): Comparison between groups according to systolic blood pressure (mmHg).

\begin{tabular}{|c|c|c|c|c|c|c|}
\hline $\begin{array}{c}\text { Systolic blood } \\
\text { pressure } \\
(\mathbf{m m H g})\end{array}$ & M0 $(\mathbf{n = 2 5})$ & $\mathbf{M 2}(\mathbf{n = 2 5})$ & $\mathbf{M 4}(\mathbf{n = 2 5})$ & M6 $(\mathbf{n = 2 5})$ & ANOVA & P-value \\
\hline Baseline & $119.48 \pm 5.97$ & $120.67 \pm 6.03$ & $118.29 \pm 5.91$ & $121.83 \pm 6.09$ & 0.137 & $>0.05$ \\
\hline After 1min. & $90.76 \pm 5.85$ & $119.47 \pm 5.97^{\mathrm{a}}$ & $117.1 \pm 5.86^{\mathrm{a}}$ & $120.62 \pm 6.03^{\mathrm{a}}$ & 7.535 & $<0.001^{* *}$ \\
\hline After 3min. & $85.12 \pm 5.02$ & $112.3 \pm 5.62^{\mathrm{a}}$ & $111.25 \pm 5.56^{\mathrm{a}}$ & $114.58 \pm 5.73^{\mathrm{a}}$ & 6.782 & $<0.001^{* *}$ \\
\hline After 5min. & $95.01 \pm 4.23$ & $103.32 \pm 5.17^{\mathrm{a}}$ & $103.46 \pm 5.17^{\mathrm{a}}$ & $105.42 \pm 5.27^{\mathrm{a}}$ & 6.103 & $<0.001^{* *}$ \\
\hline After 10min. & $100.47 \pm 4.10$ & $85.75 \pm 4.29^{\mathrm{a}}$ & $88.98 \pm 4.45^{\mathrm{a}}$ & $89.61 \pm 4.48^{\mathrm{a}}$ & 6.042 & $<0.001^{* *}$ \\
\hline After 15min. & $100.85 \pm 3.47$ & $88.32 \pm 4.42^{\mathrm{a}}$ & $91.64 \pm 4.58^{\mathrm{a}}$ & $92.29 \pm 4.61^{\mathrm{a}}$ & 5.438 & $0.004^{*}$ \\
\hline After 20min. & $109.81 \pm 5.49$ & $110.41 \pm 5.52$ & $112.72 \pm 5.64$ & $113.52 \pm 5.68$ & 0.177 & $>0.05$ \\
\hline After 25min. & $109.92 \pm 5.50$ & $110.52 \pm 5.53$ & $112.84 \pm 5.64$ & $113.63 \pm 5.68$ & 0.148 & $>0.05$ \\
\hline After 30min. & $110.03 \pm 5.50$ & $110.63 \pm 5.53$ & $113.96 \pm 5.70$ & $113.75 \pm 5.69$ & 0.542 & $>0.05$ \\
\hline After 35min. & $110.14 \pm 5.51$ & $112.84 \pm 5.64$ & $116.24 \pm 5.81$ & $113.86 \pm 5.69$ & 0.330 & $>0.05$ \\
\hline After 40min. & $112.34 \pm 5.62$ & $112.95 \pm 5.65$ & $116.36 \pm 5.82$ & $113.98 \pm 5.70$ & 0.330 & $>0.05$ \\
\hline After 45min. & $113.47 \pm 5.67$ & $114.08 \pm 5.70$ & $116.48 \pm 5.82$ & $115.12 \pm 5.76$ & 1.308 & $>0.05$ \\
\hline After 50min. & $113.58 \pm 5.68$ & $114.31 \pm 5.72$ & $117.64 \pm 5.88$ & $115.23 \pm 5.76$ & 0.330 & $>0.05$ \\
\hline After 55min. & $113.69 \pm 5.68$ & $114.42 \pm 5.72$ & $117.76 \pm 5.89$ & $117.54 \pm 5.88$ & 0.168 & $>0.05$ \\
\hline After 60 min. & $113.81 \pm 5.69$ & $114.65 \pm 5.73$ & $117.88 \pm 5.89$ & $117.65 \pm 5.88$ & 2.110 & $>0.05$ \\
\hline * Significant. ** Highly significant
\end{tabular}

* Significant. ** Highly significant

a: Significant difference with M0 $(\mathrm{p}<0.05)$ 
Table (3): Comparison between groups according to diastolic blood pressure (mmHg).

\begin{tabular}{|c|r|r|r|r|c|}
\hline $\begin{array}{c}\text { Diastolic blood } \\
\text { pressure } \\
\text { (mmHg) }\end{array}$ & M0 (n=25 & M2 $(\mathbf{n = 2 5})$ & M4 (n=25) & M6 (n=25) & P-value \\
\hline Baseline & $72.14 \pm 5.7$ & $73.14 \pm 5.85$ & $71.76 \pm 5.74$ & $74.11 \pm 5.93$ & $>0.05$ \\
\hline After 1min. & $60.85 \pm 5.6$ & $72.41 \pm 5.79$ & $71.04 \pm 5.68$ & $73.37 \pm 5.87$ & $<0.001^{* *}$ \\
\hline After 3min. & $52.09 \pm 2.3$ & $68.06 \pm 5.44$ & $67.49 \pm 5.40$ & $69.7 \pm 5.58^{\mathrm{a}}$ & $<0.001^{* *}$ \\
\hline After 5min. & $53.69 \pm 4.4$ & $56.49 \pm 4.52$ & $56.02 \pm 4.48$ & $57.85 \pm 4.63$ & $0.034^{*}$ \\
\hline After 10min. & $55.66 \pm 3.6$ & $46.89 \pm 3.75$ & $48.17 \pm 3.85$ & $49.17 \pm 3.93$ & $0.025^{*}$ \\
\hline After 15min. & $60.03 \pm 2.7$ & $48.3 \pm 3.86^{2}$ & $49.62 \pm 3.97$ & $50.65 \pm 4.05$ & $0.011^{*}$ \\
\hline After 20min. & $61.14 \pm 4.8$ & $60.37 \pm 4.83$ & $61.03 \pm 4.88$ & $62.3 \pm 4.98$ & $>0.05$ \\
\hline After 25min. & $61.21 \pm 4.9$ & $60.43 \pm 4.83$ & $61.09 \pm 4.89$ & $62.36 \pm 4.99$ & $>0.05$ \\
\hline After 30min. & $61.27 \pm 4.9$ & $60.49 \pm 4.84$ & $61.7 \pm 4.94$ & $62.42 \pm 4.99$ & $>0.05$ \\
\hline After 35min. & $61.33 \pm 4.9$ & $61.7 \pm 4.94$ & $62.94 \pm 5.04$ & $62.49 \pm 5.00$ & $>0.05$ \\
\hline After 40min. & $61.39 \pm 4.9$ & $61.76 \pm 4.94$ & $63 \pm 5.04$ & $62.55 \pm 5.00$ & $>0.05$ \\
\hline After 45min. & $61.45 \pm 4.9$ & $62.38 \pm 4.99$ & $63.0 \pm 65.04$ & $63.8 \pm 5.10$ & $>0.05$ \\
\hline After 50min. & $61.51 \pm 4.9$ & $62.5 \pm 5.00$ & $63.69 \pm 5.10$ & $63.86 \pm 5.11$ & $>0.05$ \\
\hline After 55min. & $61.57 \pm 4.9$. & $62.57 \pm 5.01$ & $63.76 \pm 5.10$ & $64.5 \pm 5.16$ & $>0.05$ \\
\hline After 60min. & $61.64 \pm 4.9$. & $62.69 \pm 5.02$ & $63.82 \pm 5.11$ & $64.57 \pm 5.17$ & $>0.05$ \\
\hline
\end{tabular}

* Significant. ** Highly significant

a: Significant difference with M0 $(\mathrm{p}<0.05)$

Table (4): Comparison between groups according to mean arterial blood pressure (mmHg).

\begin{tabular}{|c|c|c|c|c|c|}
\hline $\begin{array}{c}\text { Mean Arterial } \\
\text { Blood } \\
\begin{array}{c}\text { Pressure } \\
\text { (mmHg) }\end{array}\end{array}$ & M0 (n=25) & M2 $(\mathbf{n = 2 5})$ & M4 (n=25) & M6 (n=25) & P-value \\
\hline Baseline & $87.92 \pm 6.15$ & $88.98 \pm 6.23$ & $87.27 \pm 6.11$ & $90.02 \pm 6.30$ & $>0.05$ \\
\hline After 1 minute. & $70.08 \pm 6.03$ & $88.10 \pm 6.17^{\mathrm{a}}$ & $86.39 \pm 6.05^{\mathrm{a}}$ & $89.12 \pm 6.24^{\mathrm{a}}$ & $<0.001^{* *}$ \\
\hline After 3min. & $62.77 \pm 5.72$ & $82.81 \pm 5.80^{\mathrm{a}}$ & $82.08 \pm 5.75^{\mathrm{a}}$ & $84.66 \pm 5.93^{\mathrm{a}}$ & $<0.001^{* *}$ \\
\hline After 5min. & $66.46 \pm 3.93$ & $72.10 \pm 5.05^{\mathrm{a}}$ & $71.83 \pm 5.03^{\mathrm{a}}$ & $73.71 \pm 5.16^{\mathrm{a}}$ & $0.041^{*}$ \\
\hline After 10min. & $70.78 \pm 4.04$ & $59.84 \pm 4.19^{\mathrm{a}}$ & $61.77 \pm 4.32^{\mathrm{a}}$ & $62.65 \pm 4.39^{\mathrm{a}}$ & $0.019^{*}$ \\
\hline After 15min. & $71.51 \pm 3.17$ & $61.64 \pm 4.31^{\mathrm{a}}$ & $63.63 \pm 4.45^{\mathrm{a}}$ & $64.53 \pm 4.52^{\mathrm{a}}$ & $0.024^{*}$ \\
\hline After 20min. & $77.36 \pm 5.42$ & $77.05 \pm 5.39$ & $78.26 \pm 5.48$ & $79.37 \pm 5.56$ & $>0.05$ \\
\hline After 25min. & $77.45 \pm 5.42$ & $77.13 \pm 5.40$ & $78.34 \pm 5.48$ & $79.45 \pm 5.56$ & $>0.05$ \\
\hline After 30min. & $77.52 \pm 5.43$ & $77.20 \pm 5.40$ & $79.12 \pm 5.54$ & $79.53 \pm 5.57$ & $>0.05$ \\
\hline After 35min. & $77.60 \pm 5.43$ & $78.75 \pm 5.51$ & $80.71 \pm 5.65$ & $79.61 \pm 5.57$ & $>0.05$ \\
\hline After 40min. & $78.37 \pm 5.49$ & $78.82 \pm 5.52$ & $80.79 \pm 5.66$ & $79.69 \pm 5.58$ & $>0.05$ \\
\hline After 45min. & $78.79 \pm 5.52$ & $79.61 \pm 5.57$ & $80.83 \pm 5.66$ & $80.91 \pm 5.66$ & $>0.05$ \\
\hline After 50min. & $78.87 \pm 5.52$ & $79.77 \pm 5.58$ & $81.67 \pm 5.72$ & $80.98 \pm 5.67$ & $>0.05$ \\
\hline After 55min. & $78.94 \pm 5.53$ & $79.85 \pm 5.59$ & $81.76 \pm 5.72$ & $82.18 \pm 5.75$ & $>0.05$ \\
\hline After 60min. & $79.03 \pm 5.53$ & $80.01 \pm 5.60$ & $81.84 \pm 5.73$ & $82.26 \pm 5.76$ & $>0.05$ \\
\hline
\end{tabular}

* Significant. ** Highly significant

a: Significant difference with M0 $(\mathrm{p}<0.05)$

There were statistically significant difference between (M0) group and other groups (M2, M4 and M6) as regard heart rate from $1 \mathrm{~min}$. to $15 \mathrm{~min}$. after induction of spinal anesthesia and there were no statistically significant difference between groups from $15 \mathrm{~min}$ up to end of the surgery as in table (5). 
Table (5): Comparison between groups according to heart rate (Beat/min).

\begin{tabular}{|c|l|l|l|l|l|}
\hline $\begin{array}{l}\text { Heart rate } \\
\text { (Beat/min) }\end{array}$ & M0 $(\mathbf{n = 2 5})$ & M2 $(\mathbf{n = 2 5})$ & M4 $(\mathbf{n = 2 5})$ & M6 $(\mathbf{n = 2 5})$ & P-value \\
\hline Baseline & $86.16 \pm 4.31$ & $87.33 \pm 4.37$ & $85.87 \pm 4.29$ & $88.04 \pm 4.40$ & $>0.05$ \\
\hline After 1min. & $91.12 \pm 35.14$ & $86.46 \pm 10.32^{\mathrm{a}}$ & $85.01 \pm 10.25^{\mathrm{a}}$ & $86.56 \pm 10.36^{\mathrm{a}}$ & $0.024^{*}$ \\
\hline After 3min. & $110.13 \pm 30.01$ & $81.27 \pm 12.06^{\mathrm{a}}$ & $80.76 \pm 8.04^{\mathrm{a}}$ & $82.82 \pm 8.14^{\mathrm{a}}$ & $<0.001^{* *}$ \\
\hline After 5min. & $100.33 \pm 20.97$ & $80.7 \pm 10.04^{\mathrm{a}}$ & $80.19 \pm 10.01^{\mathrm{a}}$ & $82.04 \pm 9.10^{\mathrm{a}}$ & $<0.001^{* *}$ \\
\hline After 10min. & $90.54 \pm 20.93$ & $80.14 \pm 20.01^{\mathrm{a}}$ & $79.63 \pm 20.98^{\mathrm{a}}$ & $81.16 \pm 15.06^{\mathrm{a}}$ & $0.006^{*}$ \\
\hline After 15min. & $90.75 \pm 10.89$ & $79.58 \pm 15.98^{\mathrm{a}}$ & $79.08 \pm 13.95^{\mathrm{a}}$ & $81.02 \pm 10.05^{\mathrm{a}}$ & $0.002^{*}$ \\
\hline After 20min. & $88.53 \pm 10.93$ & $80.25 \pm 10.01$ & $79.75 \pm 10.99$ & $81.21 \pm 8.06$ & $>0.05$ \\
\hline After 25min. & $90.31 \pm 10.97$ & $80.93 \pm 8.05$ & $80.43 \pm 8.02$ & $82.04 \pm 9.10$ & $>0.05$ \\
\hline After 30min. & $85.11 \pm 10.01$ & $81.62 \pm 9.08$ & $81.11 \pm 9.06$ & $82.62 \pm 7.13$ & $>0.05$ \\
\hline After 35min. & $86.91 \pm 8.05$ & $82.32 \pm 6.12$ & $81.82 \pm 4.09$ & $83.18 \pm 5.16$ & $>0.05$ \\
\hline After 40min. & $85.83 \pm 5.04$ & $82.23 \pm 10.11$ & $81.72 \pm 7.09$ & $83.10 \pm 4.16$ & $>0.05$ \\
\hline After 45min. & $83.75 \pm 4.04$ & $82.15 \pm 5.11$ & $81.64 \pm 4.08$ & $83.12 \pm 4.16$ & $>0.05$ \\
\hline After 50min. & $84.83 \pm 4.04$ & $82.23 \pm 4.11$ & $81.72 \pm 4.09$ & $83.3 \pm 4.17$ & $>0.05$ \\
\hline After 55min. & $81.63 \pm 4.08$ & $82.93 \pm 4.15$ & $82.41 \pm 4.12$ & $83.97 \pm 4.20$ & $>0.05$ \\
\hline After 60min. & $82.45 \pm 4.12$ & $83.64 \pm 4.18$ & $83.11 \pm 4.16$ & $84.64 \pm 4.23$ & $>0.05$ \\
\hline
\end{tabular}

* Significant. ** Highly significant

a: Significant difference with M0 ( $<<0.05)$.

There were no statistically significant difference between groups according to onset of motor block at 1 and 3 minutes after induction of spinal anesthesia as in table (6).

Table (6): Comparison between groups according to onset of motor block (modified Bromage score).

\begin{tabular}{|c|c|c|c|c|c|}
\hline $\begin{array}{c}\text { Onset of motor block } \\
\text { (Modified Bromage score) }\end{array}$ & $\begin{array}{c}\text { M0 } \\
(\mathbf{n = 2 5})\end{array}$ & $\begin{array}{c}\text { M2 } \\
(\mathbf{n = 2 5})\end{array}$ & $\begin{array}{c}\text { M4 } \\
(\mathbf{n = 2 5})\end{array}$ & $\begin{array}{c}\text { M6 } \\
(\mathbf{n = 2 5})\end{array}$ & $\begin{array}{c}\text { P- } \\
\text { value }\end{array}$ \\
\hline At 1min. & $2(100 \%)$ & $2(100 \%)$ & $2(100 \%)$ & $2(100 \%)$ & $>0.05$ \\
\hline At 3min. & $3(100 \%)$ & $3(100 \%)$ & $3(100 \%)$ & $3(100 \%)$ & $>0.05$ \\
\hline
\end{tabular}

There was no statistically significant difference between groups according to Apgar score at 1 and 5 minutes after delivery as in table (7).

Table (7): Comparison between groups according to Apgar score.

\begin{tabular}{|c|c|c|c|c|c|}
\hline Apgar score & $\begin{array}{c}\text { M0 } \\
(n=25)\end{array}$ & $\begin{array}{c}\text { M2 } \\
(\mathrm{n}=25)\end{array}$ & $\begin{array}{c}\text { M4 } \\
(\mathrm{n}=25)\end{array}$ & $\begin{array}{c}\text { M6 } \\
(\mathrm{n}=25) \\
\end{array}$ & P-valu \\
\hline $\begin{array}{c}\text { At } 1 \text { min. } \\
\text { Range } \\
\text { Median (IQR) }\end{array}$ & $\begin{array}{c}6-8 \\
7(2)\end{array}$ & $\begin{array}{c}7-9 \\
8(1)\end{array}$ & $\begin{array}{c}7-9 \\
8(1)\end{array}$ & $\begin{array}{c}7-9 \\
8(1)\end{array}$ & $>0.05$ \\
\hline $\begin{array}{c}\text { At } 5 \text { min. } \\
\text { Range } \\
\text { Median (IQR) }\end{array}$ & $\begin{array}{l}9-10 \\
9(1)\end{array}$ & $\begin{array}{l}9-10 \\
9(1)\end{array}$ & $\begin{array}{l}9-10 \\
9(1)\end{array}$ & $\begin{array}{l}9-10 \\
9(1)\end{array}$ & $>0.05$ \\
\hline
\end{tabular}

H-Kruskal Wallis-H test;

There was statistically significant difference between group M0 and other groups according to Rescue analgesia (regression of sensory block to level T12) "min". The regression of sensory block to level T12 and patient need analgesia was longer in group M0 than other groups and was faster in group M6 and there were no statistically significant difference between groups M2 and M4 as in table (8).

Table (8): Comparison between groups according to rescue analgesia

\begin{tabular}{|c|c|c|c|c|c|}
\hline $\begin{array}{l}\text { Rescue analgesi } \\
\text { (regression of } \\
\text { sensory block t } \\
\text { level T12) "min }\end{array}$ & M0 $(n=25$ & M2 $(n=25)$ & M4 $(n=25)$ & M6 $(n=25)$ & P-valur \\
\hline Mean \pm SD & $119.6 \pm 21.1$ & $109.2 \pm 23.32$ & $110.24 \pm 21.2$ & $104.0 \pm 15.9^{\mathrm{ab}}$ & $0.012 *$ \\
\hline
\end{tabular}

a: Significant difference between M0 and M2

b: Significant difference between M4 and M0

abc: Significant difference between M6 and other groups. 
There were statistically significant difference between all groups according to motor block regression (time of Bromage score 2 "min"). There were longer time in group M6 than other groups as in table (9).

Table (9): Comparison between groups according to motor block regression (time of Bromage score 2 "min".

\begin{tabular}{|c|r|c|c|c|c|}
\hline $\begin{array}{c}\text { Motor block regression } \\
\text { (time of Bromage score 2 "min") }\end{array}$ & M0 (n=25) & M2 $(\mathbf{n = 2 5})$ & M4 (n=25) & M6 (n=25) & P-value \\
\hline Mean \pm SD & $156.45 \pm 21.20$ & $166.88 \pm 26.50 *$ & $177.31 \pm 21.20 *$ & $187.74 \pm 31.80^{*}$ & $0.004 *$ \\
\hline
\end{tabular}

*: Significant difference between all groups.

As regard nausea and vomiting: There was statistically significant difference between group M0 and other groups (M2, M4 and M6) as in table (10). As regard Bradycardia: There was statistically significant difference between all groups as in table (10). As regard Pruritus: There was no statistically significant difference between all groups as in table (10).

Table (10): Comparison between groups according to complications.

\begin{tabular}{|l|l|l|l|l|l|}
\hline Complications & M0 $(\mathbf{n = 2 5})$ & M2 $(\mathbf{n = 2 5})$ & $\mathbf{M 4}(\mathbf{n = 2 5})$ & M6 $(\mathbf{n = 2 5})$ & P-value \\
\hline Nausea and vomiting & $9(36 \%)$ & $5(20 \%) \mathrm{a}$ & $4(16 \%) \mathrm{a}$ & $4(16 \%) \mathrm{a}$ & 0.038 \\
\hline Bradycardia & $9(36 \%)^{*}$ & $5(20 \%)^{*}$ & $4(16 \%)^{*}$ & $2(8 \%)^{*}$ & 0.038 \\
\hline Pruritus & $3(12 \%)$ & $2(8 \%)$ & $2(8 \%)$ & $3(12 \%)$ & $>0.05$ \\
\hline
\end{tabular}

a: Significant difference with M0 ( $\mathrm{p}<0.05)$ *: Significant difference between all groups.

\section{DISCUSSION}

Hypotension is one of the most common complications of spinal anesthesia in women undergoing cesarean section. In a number of studies, the effect of patient positioning during or after spinal anesthesia has been studied, although conflicting results have been reported ${ }^{(1)}$.

In the current study we used sitting position during induction of spinal anesthesia because it was easier for anesthetic administration and there was no difference between sitting position and another position as lateral position on hemodynamic variables and this was approved by the study done by Chevuri et al. which reported that the only advantage of the sitting position, compared to the lateral posture, was easier anesthetic administration. Also, similar hemodynamic stability, quality of analgesia, and muscle relaxation were found in the investigation by Chevuri et al. ${ }^{(7)}$. Also, Ortiz-Gómez et al. studied the sitting, left, and right lateral decubitus positions during spinal anesthesia induction with hyperbaric bupivacaine plus $20 \mu \mathrm{g}$ of fentanyl. Although the incidence of hypotension and vasopressor requirements did not vary significantly, the sitting position was recommended, as it was easier to administer the anesthetic and was more comfortable for the patients ${ }^{(3)}$.

Moreover, in a study by Kharge $\boldsymbol{e t}$ al. inducing position for spinal anesthesia did not affect hemodynamic stability and block characteristics in both the sitting and lateral positions during induction of spinal anesthesia except that patients feel more comfortable in lateral position ${ }^{(8)}$. In another study by Wang $\boldsymbol{e t}$ al. the incidence of hypotension and ephedrine requirements in patients who remained in the left lateral position was lower than patients shifted to a left-tilt supine posture after spinal anesthesia in cesarean section ${ }^{(9)}$. And in study of Simin et al. the changes in maternal hemodynamic were significantly lower in lateral position than sitting position in patients undergoing spinal anesthesia for cesarean section ${ }^{(\mathbf{1 0})}$.

In the current study the incidence of hypotension was decreased in setting groups versus immediately lying down group where the frequency of hypotension in group M0 (lying down immediately) was (84\%) while in group M2 which sat for 2 minutes was $(52 \%)$, in group M4 which sat for 4 minutes was (36\%), and in group M6 which sat for 6 minutes was $(24 \%)$.

We found that the frequency of hypotension in the first 5 minutes after spinal anesthesia was significantly lower in women who remained seated for 2, 4, and 6 minute compared to those who were immediately placed in a supine position after spinal anesthesia. Although hypotension occurred at 10 or 15 minute after spinal anesthesia in sitting groups, but it was less frequent than immediately lying down group, this hypotension occurred at SBP, DBP, and MAP.

This result was supported by a previous study of Hajian et al., who found that sitting up for 1 and 2 minutes rather than immediately lying down lowered the frequency of hypotension where the frequency of hypotension in group which was lying down immediately was $(92 \%)$ while in group which sat for 1 minutes was $(66 \%)$, and in group which sat for 2 minutes was $(50 \%){ }^{(11)}$.

Also this result was supported by study of Agrawal and Rawlani, who found that keeping the patient in sitting position for 30 seconds helps to prevent high spinal and gives better hemodynamic stability ${ }^{(12)}$.

Gori et al. in similar studies found that sitting up for 3 and 2 minutes, respectively did not influence the incidence of maternal hypotension versus immediately lying down. The varying doses and 
baricities of bupivacaine, used for spinal anesthesia, might have resulted in the discrepancies between these studies and the present research. Also, use of isobaric bupivacaine in the study by Gori $\boldsymbol{e t} \boldsymbol{a l}$. in comparison with hyperbaric bupivacaine in the present study, as well as the higher dose of bupivacaine could explain these conflicting results ${ }^{(13)}$.

In this study the total dose of the required ephedrine was lower in the sitting groups and there was significant difference between sitting groups where group M6 was lower than group M4 and M2 and group M4 was lower than group M2, this mean that when patient sits after induction of spinal anesthesia for long time, this will decrease the incidence of using ephedrine and total dose of it.

Also, in this study the total dose of the required fluid was lower in the sitting groups rather than immediately lying down and the required atropine was higher in group M0 than sitting groups and there were no significant difference between sitting group and each other.

This result was supported by Sargin et al., who found that sitting up for 1 and 3 minutes rather than immediately lying down lowers the total dose of the required ephedrine, atropine and the total required fluid (14). Also this result was supported by El-Hakeem $\boldsymbol{e t}$ al . (6), who found that sitting up for 5 minutes rather than immediately lying down lowers the total dose of the required ephedrine, atropine and the total required fluid (6).

The study done by El-Hakeem et al. (6), reported that sitting the patient up for up to $7 \mathrm{~min}$ after combined spinal and epidural anesthesia (CSE) for cesarean section reduced intraoperative ephedrine requirement without affecting the success of the spinal anesthetic.

While Gori $\boldsymbol{e t}$ al. in similar study found that sitting up for 3 and 2 minutes, respectively did not influence the total dose of the required ephedrine, atropine and the total required fluid versus immediately lying down ${ }^{(\mathbf{1 3})}$.

In the current study the onset of sensory block was delayed in subjects who sat for 2, 4 and 6 minutes after spinal anesthesia than who were immediately lying down and there were significant difference between sitting groups; where in group M6 the onset of sensory block was delayed than group M4 and M2 and group M4 was delayed than group M2. This mean that when patient sits after induction of spinal anesthesia for long time, this will delay the onset of sensory block. Although there were some cases from group M6 didn`t reach up to T6 sensory level block and needed conversion to general anesthesia but in other groups (M0, M2 and M4) there were no need to conversion to general anesthesia because their sensory level block was adequate.

On the other hand, there were no statistically significant difference between the groups according to onset of motor block at 1 and 3 minutes after induction of spinal anesthesia because all groups reached Bromage scale of 2 at 1 minute and reached to a Bromage scale of 3 at 3 minutes.

This result was supported by study of Sargin $\boldsymbol{e t}$ al. where the onset of sensory block was delayed respectively, in subjects who seated for 1 and 3 minutes with no need to conversion to general anesthesia because their sensory level block was adequate in all groups ${ }^{(14)}$. Also this result supported by Hajian et al. (11) and El-Hakeem et al. ${ }^{(6)}$ where the onset of sensory block was delayed respectively, in subjects who seated for 1,2 and 5 minutes but there were no need to conversion to general anesthesia because their sensory level block was adequate in all groups ${ }^{(6,11)}$. Gori et al. reported that delayed supine positioning didn't influence dermatomal spread of anesthesia but the level of anesthesia spread to dermatomal levels was higher in group which immediately were lying down than sitting groups but there were complete motor block of the lower limbs ${ }^{(\mathbf{1 3})}$.

In the present study there were no statistically significant difference between groups according to Apgar score at 1 and 5 minutes after delivery because all groups recorded Apgar score range from 7 to 9 at 1 minute after delivery and range from 9 to 10 at 5 minutes after delivery and according to urine output there were no statistically significant difference between groups. This was supported by Hajian $\boldsymbol{e t}$ al . and Sargin et $\boldsymbol{a l}$. while there were statistically significant difference between groups according to total blood loss as there was less blood loss in group M0 than sitting groups with no difference between sitting groups and each other but this was opposed by Hajian et al. and Sargin et al. because there was no statistically significant difference between groups according to blood loss ${ }^{(11,14)}$.

The study that was done by El-Hakeem $\boldsymbol{e t}$ al. (6) reported that Apgar score were better in group which sat for 5 minutes than group which was immediately lying down at 1 minute after birth. However, there was no difference between groups at 5 minutes after birth and the estimated intraoperative blood loss was slightly higher in the immediately lying down group ${ }^{(6)}$.

In this study the incidence of nausea and vomiting was decreased in sitting groups rather than immediately lying down but there was no statistically significant difference between groups as regard pruritus. This was supported by El-Hakeem $\boldsymbol{e t}$ al. ${ }^{(6)}$ who reported that the increased occurrence of nausea and vomiting among group which was immediately lying down. This could be explained by the higher level of maximum sensory block achieved in this group but this was opposed by El-Hakeem $\boldsymbol{e t}$ al. and Hajian $\boldsymbol{e t}$ $a l$., who showed no statistically significant difference between groups according to nausea, vomiting and pruritus ${ }^{(6,11)}$. 
Finally, group of patients who sat for 6 minutes after spinal anesthesia had shorter duration in regression of sensory block to level T12 than other groups and this time which patient need analgesia but this group had longer duration in regression of motor block according to Bromage score 2 than other groups while group (M0) who immediately laying down after spinal anesthesia had longer duration in regression of sensory block to level T12 than other groups and this time which patient need analgesia but this group had shorter duration in regression of motor block according to Bromage score 2 than other groups but groups (M2 and M4) were between M6 and M0 groups. This was supported by El-Hakeem et al. ${ }^{\left({ }^{(6)}\right.}$ and Sargin et al. ${ }^{\left({ }^{(14)} \text {. }\right.}$

Although, the study of Hajian et al. reported that regression of sensory block from T6 to T10 was faster in the group which sat for 2 minutes than other groups but motor block regression time to Bromage score 2 was faster in the group which sat for 2 minutes than other groups ${ }^{(\mathbf{1 1})}$.

Considering multiple variations in the design of conducted studies, it is rather difficult to analyze the influence of maternal posture during spinal anesthesia administration. In general, different local anesthetic doses, different baricities and adjuvants, and various maternal positions have been assessed.

In general, keeping the patient upright for several minutes after spinal anesthesia leads to delayed aortocaval compression and limited spread of the local anesthetic. This in fact might be the cause of the reduced incidence of hypotension and longer time to achieve T6 sensory level due to more stable hemodynamics in the first few minutes after subarachnoid block ${ }^{(6)}$.

\section{CONCLUSION}

The present study revealed that the patient's position is an important factor that affects the frequency of hypotension, ephedrine and fluid requirements, and the onset of sensory block during the administration of spinal anesthesia for cesarean section. Based on the findings, keeping the parturient seated for 2, 4 or 6 minutes after spinal anesthesia, compared to immediately lying down, could decrease the frequency of hypotension in the first 5 minutes before delivery and also ephedrine and total fluid required during operation. Although this hypotension occurred at 10 or 15 minute after spinal anesthesia in sitting groups, it is less frequent than immediately lying down group. $12 \%$ of the parturient who seated for 6 minutes after spinal anesthesia had no adequate sensory level block and needed conversion to general anesthesia.

\section{REFERENCES}

1. Hingson RA, Hellman LM (2015): In: Miller's Anesthesia. Hingson RA, Hellman LM, editors. Philadelphia: Saunders; Anesthesia for Obstetrics. https://www.elsevier.com/books/millers-anesthesia-2volume-set/miller/978-0-7020-5283-5

2. Mitra JK, Royal J, Bhattachharyya PM et al. (2013): Changing trends in the management of hypotension following spinal anesthesia in cesarean section. Journal of Postgraduate Medicine, 59(2): 121.

3. Ortiz-Gómez JR, Palacio-Abizanda FJ, MorillasRamirez F et al. (2015): Effect of position on maternal haemodynamics during elective caesarean delivery under spinal anaesthesia. Anaesthesia, 5: 7-9.

4. Mercier FJ, Augè M, Hoffmann C et al. (2013): Maternal hypotension during spinal anesthesia for caesarean delivery. Minerva Anestesiol., 79(1): 62-73.

5. Obasuyi BI, Fyneface-Ogan S, Mato CN (2013): A comparison of the haemodynamic effects of lateral and sitting positions during induction of spinal anaesthesia for caesarean section. International journal of obstetric anesthesia, 22(2): 124-128.

6. El-Hakeem EE, Kaki AM, Almazrooa AA et al. (2011): Effects of sitting up for five minutes versus immediately lying down after spinal anesthesia for Cesarean delivery on fluid and ephedrine requirement; a randomized trial. Canadian Journal of Anesthesia, 58(12): 1083-1089.

7. Chevuri SB, Rao JS, Chandergutti V et al. (2015): A Comparative Study of Effects of Sitting and Lateral Positions on Quality of Block during Induction of Spinal Anaesthesia in Patients Undergoing Cesarean Section. J Cont Med A Dent., 3(1): 93-4.

8. Kharge ND, Mali A, Gujjar P (2017): Comparison of haemodynamic effects of lateral and sitting positions during induction of spinal anesthesia for elective caesarean section. Int J Res Med Sci., 5: 851-6.

9. Wang X, Xu JM, Zhou F et al. (2015): Maternal position and development of hypotension in patients undergoing cesarean section under combined spinal-epidural anesthesia of intrathecal hyperbaric ropivacaine. International Medical Journal of Experimental and Clinical Research, 21: 52-55.

10. Simin A, Naghipour B, Hojjat P et al. (2018): Effect of Position during Induction of Spinal Anaesthesia for Caesarean Section on Maternal Haemodynamic: Randomised Clinical Trial. Journal of Clinical \& Diagnostic Research, 12(2): 5-8.

11. Hajian P, Nikooseresht $M$, Lotfi $T$ (2017): Comparison of 1-and 2-Minute Sitting Positions Versus Immediately Lying Down on Hemodynamic Variables After Spinal Anesthesia with Hyperbaric Bupivacaine in Elective Cesarean Section. Anesthesiology and Pain Medicine, 7(2): 36-41.

12. Agrawal N \& Rawlani S (2017): Spinal Anaesthesia in Sitting Positin for 30 Seconds Vs Conventional Spinal Anaesthesia: Which is Better? People, 10(1):17.

13. Gori F, Corradetti F, Cerotto V et al. (2010): Influence of positioning on plain levobupivacaine spinal anesthesia in cesarean section. Anesthesiology Research and Practice. https://www.hindawi.com/journals/arp/2010/212696/

14. Sargin A, Karaman S, Turksal E et al. (2016): Abstract PR200: Effects of Sitting Up for Three Minutes Or One Minute Versus Immediately Lying Down After Spinal Anesthesia for Cesarean Section. Anesthesia \& Analgesia, 123(3):

252-59. 